\title{
The Delayed Images in a Hot Rubidium Atomic Vapor
}

\author{
Long Sui \\ Changchun Institute of Optics, Fine Mechanies and Physics, Chinese Academy of Sciences, Changchun, China \\ Email:297490719@qq.com
}

Received 20 October 2015; accepted 13 November 2015; published 20 November 2015

\begin{abstract}
We report an experimental realization of the delayed images in a hot rubidium atomic vapor. With a rubidium atomic vapor cell as slow light medium, the image quality of the experiment could be improved greatly, compared with the results without a slow light medium. By analyzing the results about the image visibility of the slow light imaging system under three different conditions, the image visibility becomes better with the increment of the temperature, during the time that the wavelength of the laser is within dispersion range.
\end{abstract}

\section{Keywords}

\section{Slow Light, Rubidium Atoms, Image Visibility}

\section{Introduction}

Because the amplitude and the phase of images are important in image processing, the information should be preserved. Nowadays all-optical image processing is the more available method than others, including digital image processing, three-dimensional image processing. Although the loss still exists, the slow light technology can make the effort least. The control of velocity of light is a main research field of optical applications, such as the spectral sensitivity of interferometers using a slow light medium [1]-[3], the storage of the delayed image [4]-[7] and so on. All-optical delay of images has been realized using two Lorentzian absorption resonances in a hot cesium vapor [8]. In addition, using an image resonator [9]-[11] can also carry out the delayed images.

\section{Theoretical Analysis and Experimental Results}

In this paper, we demonstrate that the image visibility of a slow light imaging system can be gone up. The experimental setup is seen in Figure 1. The light source is a $780 \mathrm{~nm}$-wavelength tunable semiconductor laser. A binary mask plate is used as an object of the imaging experiment. An object, two lenses and a CCD camera are placed as a $4 \mathrm{f}$ imaging system. Here, the distances between the object and the first lens and between the second lens and the CCD camera, both are a focal length, and the distance between the two lenses is twifold focal lengths. In the $4 \mathrm{f}$ imaging system, a $10 \mathrm{~cm}$-length rubidium atomic vapor cell is added to the centre of two lenses. In order to observe the effect temperature had on the images, the rubidium atomic vapor cell is put in a particular place, temperature controller device. 


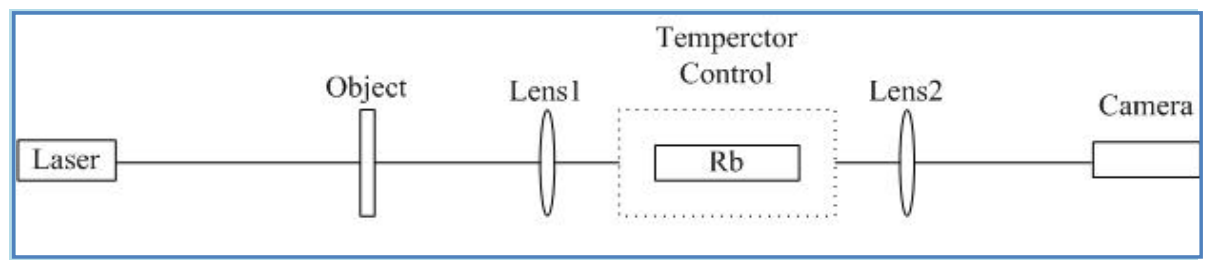

Figure 1. The schematic diagram of a slow light imaging system.

Currently, the methods of evaluation of the image quality of the optical system are divided into two aspects: First, every object can be decomposed into a combination of points. Second, every object can be decomposed into spectrums under different frequency conditions. In this data processing of the experiment, we use the first method.

We define the image visibility which is the standard of the image quality as following,

$$
V=\frac{I_{M}-I_{\mathrm{m}}}{I_{M}+I_{\mathrm{m}}},
$$

where $I_{M}$ is the maximum value of the transmission intensity and $I_{m}$ is the minimum value. The analyzed results of the experiment are separated into two aspects: on one hand, whether it has an impact on the image quality by adding rubidium atomic vapor. On the other hand, how the slow light material affects the performance of visibility of images.

Three different masks are used as objects, including circles, letters and squares. The analysis of the image quality about the rubidium atomic vapor is listed below.

We select three objects, and the image results are seen in Figure 2. In Figure 2, the image visibilities are $11.11 \%, 53.88 \%, 96.91 \%, 0 \%, 31.11 \%, 76.47 \%, 0 \%, 50.00 \%$ and $84.12 \%$, respectively, from (a) to (i). The image intensity is affected by the transmission intensity. In Equation (1), the reduction rate of numerator $I_{M}-I_{\mathrm{m}}$ is slower than the change of denominator $I_{M}+I_{m}$. Because of the rubidium absorption near the D2 transition lines ( $F=2 \rightarrow F^{\prime}=3$ ), the intensity is gone down Thus, the image intensity of a slow light imaging system is larger than that without a rubidium atomic vapor cell.

As seen in Figure 2, regardless of object, the rubidium vapor can play an important role in improving the image quality. The image visibility of the experiment within a resonant rubidium vapor is better than that off resonant frequency of rubidium vapor.

Since the rubidium absorption resonant region is very narrow, the wavelength of the laser is tuned to achieve three different demands, for example in the resonant frequency region, near the resonant frequency region and outside of the resonant frequency region. The analysis of the results is given.

The image visibilities of Figures 3(a)-(f) are 24.8\%, 99.98\%, 72.30\%, 74.67\%, 99.98\% and 13.50\%, respectively. Thus, the relationships between image visibility and temperature are drawn in Figure 3(g), Figure 3(h) and Figure 3(i). Based on the above discussions and analysis of the work, while the temperature rises, the image visibility is nicer than before, when the laser frequency is tuned inside of the resonant frequency range of the rubidium atomic vapor, in (g). However, because of the strong absorption of the light intensity, the image visibility will be reduced at the time of the temperature rising continually. It means that there is a maximum value of the image visibility when the temperature increases. In (h), since the laser frequency is near the resonant frequency of the rubidium atomic vapor region, when the temperature goes up, the changes of the image visibility are very small. In (i), the experimental data is given. Hence, it has no significant effect on the image visibility, when the wavelength of the laser is near the region of the rubidium resonance. While the laser frequency is away from the resonant frequency range of the rubidium atomic vapor, with the raising of the temperature, it is necessary that the image visibility goes down.

With temperature increasing, the population of upper-level $F^{\prime}=3$ becomes more, the rubidium absorption is stronger. The image visibility is defined by Equation (1), and it has a relationship with the transmission intensity via rubidium atomic vapor cell. When the transmission intensity is become smaller, the reduction rate of numerator $I_{M}-I_{\mathrm{m}}$ is slower than the change of denominator $I_{M}+I_{m}$. Hence, the image visibility is nicer. In other words, the temperature of the rubidium atomic vapor cell is higher, the image visibility is larger. The group index is given by $n_{g}=n+\omega \frac{d n}{d \omega}$, when the rubidium atoms are in the vicinity of the resonant frequency 


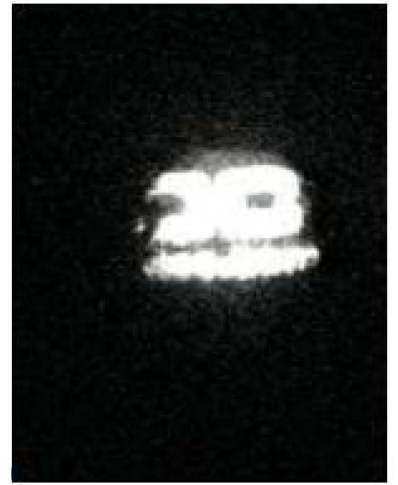

(a)

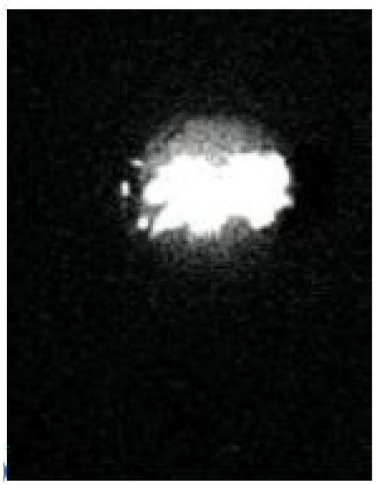

(d)

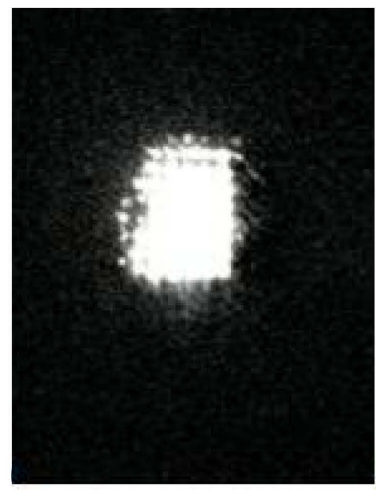

(g)

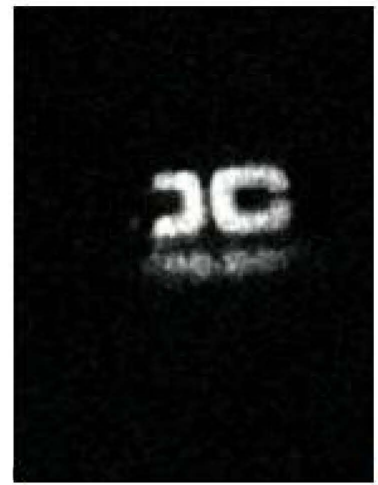

(b)

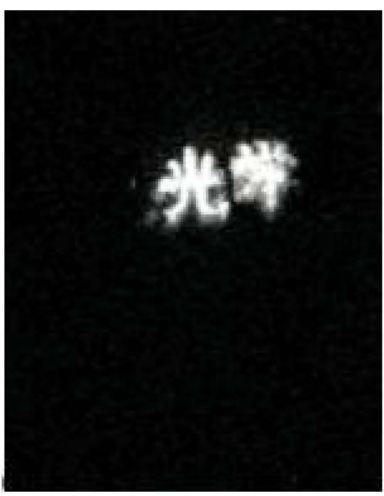

(e)

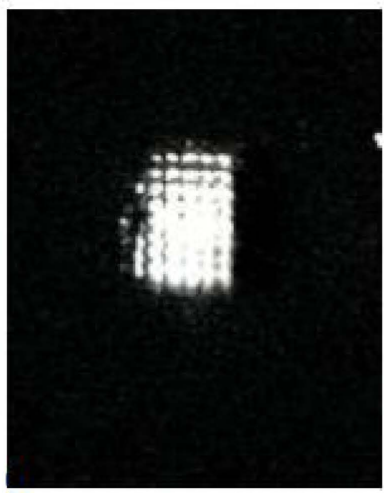

(h)

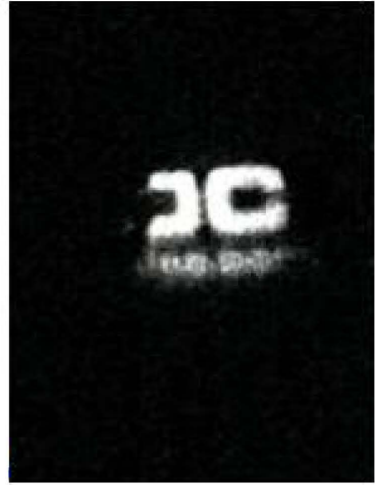

(c)

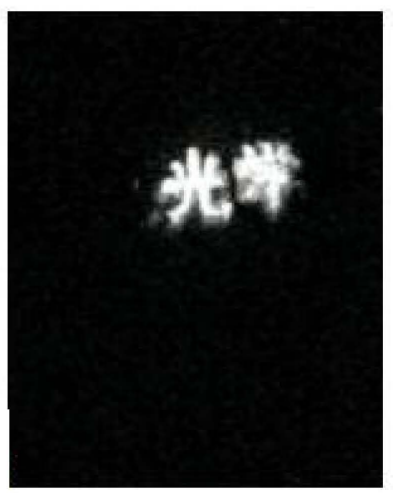

(f)

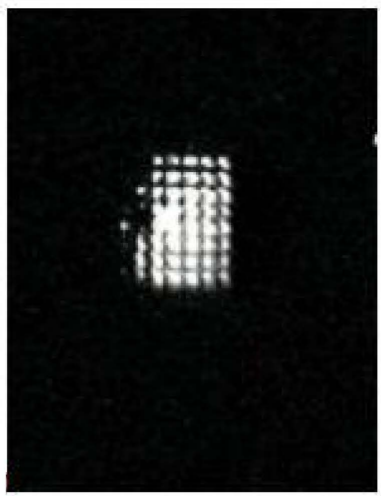

(i)

Figure 2. The image results. Select circles as objects in (a), (b), and (c); in (d), (e), and (f), objects are letters; squares are chosen as objects in (g), (h), and (i) In (a), (d) and (g), the rubidium atomic vapor cell is removed; in (b), (e) and (h), the wavelength of the laser is tuned outside of the resonant frequency; and in (c), (f) and (i), the wavelength of the laser is contained within the dispersive region.

range located between the normal dispersion and abnormal dispersion, the slope of the refractive index is zero $\left(\frac{d n}{d \omega}=0\right)$, the group index equals to the refractive index (i.e. $\left.n_{g}=n\right)$. Above all, it is an assumption that the group index of rubidium vapor is similar to the refractive index of air. The cell's temperature has few effects on improving the image visibility because the slow light imaging system can be considered as a $4 \mathrm{f}$ imaging system of the geometry optics. When the laser frequency is away from the resonant frequency region, the results of the image visibility gained are different from that of the resonant range. The reasons are the change of the refractive 


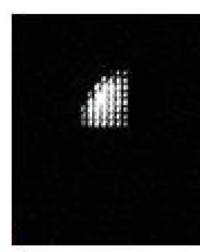

(a)

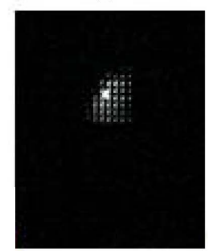

(b)

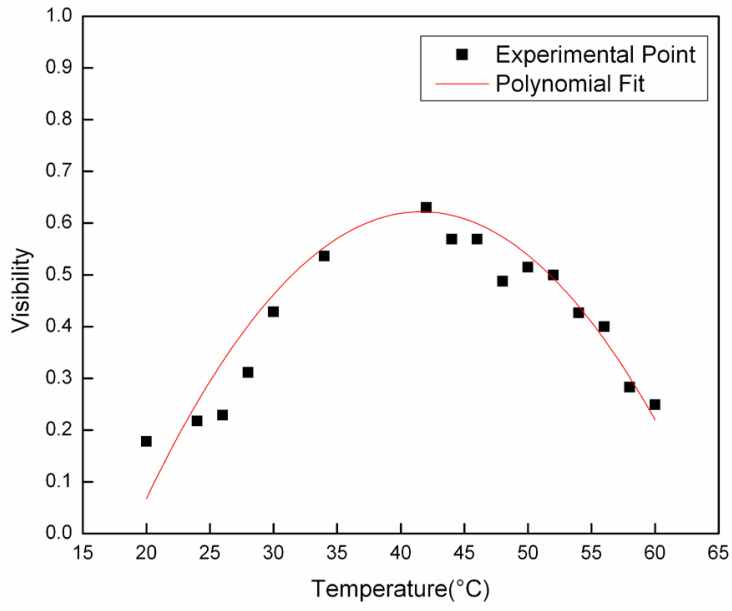

(g)

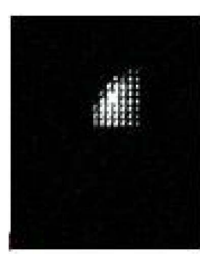

(e)

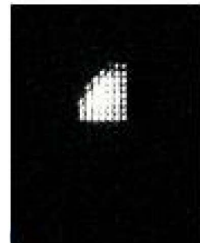

(f)

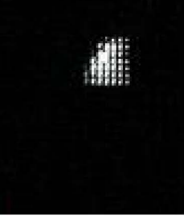

僐

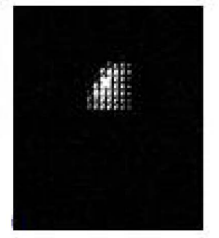

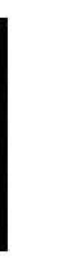

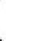

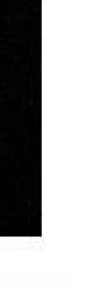

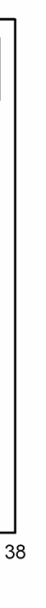

(h)

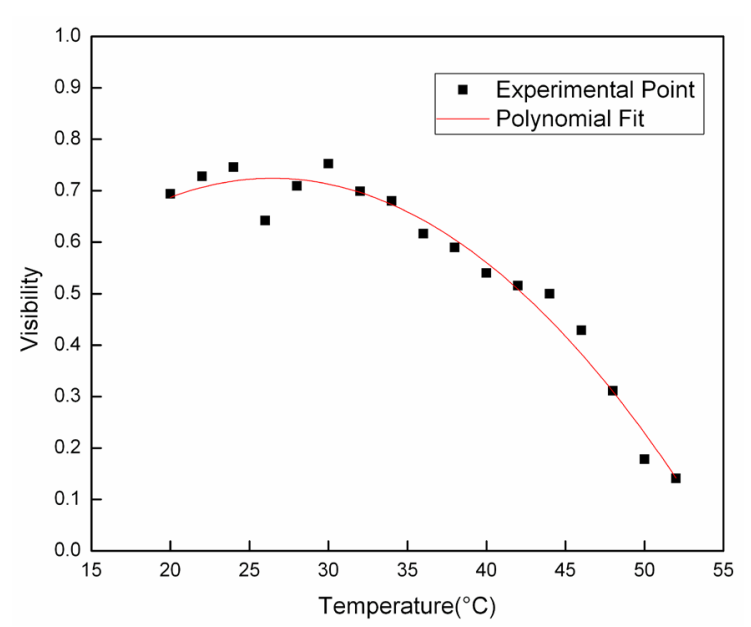

(i)

Figure 3. The results of the slow light imaging system and the analysis of images. (a), (b) and (g) is under the condition of the resonant frequency of the rubidium atomic vapor. In (c), (d) and (h), the wavelength of the laser is the near-resonant frequency of the rubidium atomic vapor. When the rubidium atomic vapor is outside of the absorption dispersion region, we obtain the image results, including (e), (f) and (i). (a) and (b) are the imaging results when temperatures are $20^{\circ} \mathrm{C}$ and $60^{\circ} \mathrm{C}$, respectively. (c) and (d) are the imaging results when temperatures are $20^{\circ} \mathrm{C}$ and $42^{\circ} \mathrm{C}$, respectively. (e) and (f) are the imaging results when temperatures are $20^{\circ} \mathrm{C}$ and $52^{\circ} \mathrm{C}$, respectively. In (g), (h) and (i), give the relationships between the temperature and the image visibility. The black points indicate experimentally measured points, and the red solid line is a polynomial fit through all the experimental points. 
index of the normal dispersive is different from that of the abnormal dispersion, and the abnormal dispersive range is the same as the resonant frequency range.

\section{Conclusion}

In conclusion, it is shown that a delayed image can be obtained in a slow light imaging system. Based on three cases above, there are several regulations: the image visibility becomes worse with the raising of the temperature, when the laser frequency is away from the resonant frequency range of rubidium. It has few effects on the image visibility by changing the temperature, while the wavelength of the laser is near the rubidium resonant region. Finally there is a maximum value of image visibility, during the time that the laser frequency is equal to the resonant frequency range of rubidium. The hot atomic vapor making images delayed can be useful in image processing, including image buffer, nonlinear imaging and image sensors.

\section{Acknowledgements}

The research is supported by the Major National Scientific Equipment Developed Project of China under Grant Nos. 2013YQ140517.

\section{References}

[1] Shi, Z.M., Boyd, R.W., Gauthier, D.J. and Dudley, C.C. (2007) Enhancing the Spectral Sensitivity of Interferometers Using Slow-Light Media. Opt. Lett., 32, 8. http://dx.doi.org/10.1364/OL.32.000915

[2] Shi, Z.M. and Boyd, R.W. (2007) Slow-Light Fourier Transform Interferometer. Phys. Rev. Lett., $99,24$. http://dx.doi.org/10.1103/PhysRevLett.99.240801

[3] Cai, Y.X., Zhang, Y.D., Yang, C.B., Dang, B.S., Wang, J.F. and Yuan, P. (2009) A High Spectral Sensitivity Interferometer Based on the Dispersive Property of the Semiconductor GaAs. Opt. Exp., 17, 24. http://dx.doi.org/10.1364/OE.17.022254

[4] Pugatch, R., Shuker, M., Firstenberg, O., Ron, A. and Davidson, N. (2007) Topological Stability of Stored Optical Vortices. Phys. Rev. Lett., 98, 20. http://dx.doi.org/10.1103/PhysRevLett.98.203601

[5] Shuker, M., Firstenberg, O., Pugatch, R., Ron, A. and Davidson, N. (2008) Storing Images in Warm Atomic Vapor. Phys. Rev. Lett., 100, 22. http://dx.doi.org/10.1103/PhysRevLett.100.223601

[6] Zhao, L., Wang, T., Xiao, Y. and Yelin, S.F. (2008) Image Storage in Hot Vapors. Phys. Rev. A., 77, 4. http://dx.doi.org/10.1103/PhysRevA.77.041802

[7] Vudyasetu, P.K., Camacho, R.M. and Howell, J.C. (2008) Storage and Retrieval of Multimode Transverse Image in Hot Atomic Rubidium Vapor. Phys. Rev. Lett., 100, 12. http://dx.doi.org/10.1103/PhysRevLett.100.123903

[8] Camacho, R.M., Broadbent, C.J., Ali-Khan, I. and Howell, J.C. (2007) All-Optical Delay of Images Using Slow Light. Phys. Rev. Lett., 98, 4. http://dx.doi.org/10.1103/PhysRevLett.98.043902

[9] Tomita, M., Sultana, P., Takami, A. and Matsumoto, T. (2010) Advanced and Delayed Images through an Image Resonator. Opt. Exp., 18, 12. http://dx.doi.org/10.1364/OE.18.012599

[10] Sultana, P., Takami, A., Matsumoto, T. and Tomita, M. (2010) Delayed Optical Images through Coupled-ResonatorInduced Transparency. Opt. Lett., 35, 20. http://dx.doi.org/10.1364/OL.35.003414

[11] Li, Q.-H. (2013) Rapid and Parallel Image Compression System with Ultra-Low Delay for Unmanned Aerial Vehicles. Optics and Precision Engineering, 21. 